\title{
Combination of Olanzapine and Samidorphan Has No Clinically Significant Effect on the Pharmacokinetics of Lithium or Valproate
}

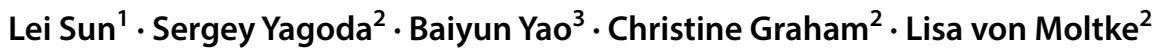

Published online: 4 October 2019

(c) The Author(s) 2019

\begin{abstract}
Background and Objective Olanzapine is an atypical antipsychotic indicated for the treatment of schizophrenia and, either as monotherapy or as an adjunct to lithium or valproate, for bipolar I disorder. Multiple daily doses of olanzapine do not affect the pharmacokinetics of lithium or valproate; therefore, concomitant olanzapine administration does not require dosage adjustment of lithium or valproate. ALKS 3831, a combination of olanzapine and the opioid receptor antagonist samidorphan (OLZ/SAM), is currently under development to provide the established antipsychotic efficacy of olanzapine while mitigating olanzapine-induced weight gain. Olanzapine is recognized as one of the most efficacious antipsychotics; however, the benefits of olanzapine are offset by its propensity to cause significant weight gain, which may lead to longterm metabolic sequelae. This study evaluated the effects of multiple daily doses of OLZ/SAM on the pharmacokinetics of lithium or valproate in healthy subjects.

Methods This was an open-label, single-sequence, two-cohort study (ALKS3831-B101) conducted at a single center in the USA. Thirty-four healthy adult subjects were assigned (1:1) to receive lithium carbonate 300-mg tablets (cohort 1) or divalproex sodium 500-mg tablets (cohort 2), once every $12 \mathrm{~h}$ on days $1-7$. Once-daily oral doses of OLZ/SAM (olanzapine $10 \mathrm{mg} / \mathrm{samidorphan} 10 \mathrm{mg}$ ) bilayer tablets were administered on days 8-18. Subjects resumed every 12-h doses of lithium or valproate concomitantly with the once-daily oral doses of OLZ/SAM on days 12-18. Plasma concentrations of lithium and valproic acid (valproate) were quantified in blood samples collected prior to and up to $12 \mathrm{~h}$ after lithium or valproate dose administration on days 7 and 18. Pharmacokinetic parameters of lithium and valproate, including maximum plasma concentration and area under the plasma concentration-time curve over a 12-h dosing interval, were calculated. The ratio of geometric means of maximum plasma concentration and area under the plasma concentration-time curve over a 12-h dosing interval in the presence and absence of OLZ/SAM, and its two-sided 90\% confidence intervals, were derived from a mixed-effects model. Adverse events were monitored throughout the study.

Results The $90 \%$ confidence intervals for the ratios of geometric means, in the presence vs. absence of OLZ/SAM, were within the equivalence interval of $80-125 \%$ for both maximum plasma concentration and area under the plasma concentration-time curve over a 12-h dosing interval of lithium and of valproate. The safety profiles of lithium or valproate coadministered with OLZ/SAM were consistent with what has been previously reported for lithium or valproate. The safety profile of OLZ/SAM was consistent with that observed in previous clinical studies with OLZ/SAM.

Conclusions Consistent with previously reported findings on olanzapine, administration of multiple doses of OLZ/SAM did not have a clinically significant effect on the pharmacokinetics of lithium or valproate. Co-administration of OLZ/SAM and lithium or valproate was generally well tolerated; the safety profile of OLZ/SAM was consistent with that observed in previous clinical studies.
\end{abstract}

\section{Introduction}

Bipolar I disorder is a chronic mental illness associated with substantial psychosocial disability, high rates of substance abuse, and a high risk of suicide [1,2]. Clinical practice guidelines recommend long-term psychiatric management of bipolar I disorder [2, 3], and pharmacotherapy remains a key component in the treatment of associated manic or mixed lei.sun@alkermes.com

Extended author information available on the last page of the article episodes [2, 4, 5]. Olanzapine is approved as monotherapy 


\section{Key Points}

OLZ/SAM, a combination of the antipsychotic olanzapine and the opioid antagonist samidorphan, is being developed to provide the established antipsychotic efficacy of olanzapine while mitigating olanzapine-induced weight gain.

Multiple daily doses of OLZ/SAM did not have a clinically significant effect on the steady-state pharmacokinetics of lithium or valproate. Therefore, OLZ/SAM can be used as an adjunct to lithium or valproate without the need for dose adjustment.

Co-administration of OLZ/SAM with lithium or valproate was generally well tolerated.

and as an adjunct to valproate or lithium [6] for the treatment of manic or mixed episodes associated with bipolar I disorder [2-5, 7, 8]. However, its use as a treatment for bipolar disorder has been limited by safety concerns related to weight gain and associated metabolic consequences [9]. ALKS 3831 is a combination of olanzapine and samidorphan (OLZ/SAM) intended to provide the established antipsychotic efficacy of olanzapine while mitigating olanzapineinduced weight gain. Olanzapine is recognized as one of the most efficacious antipsychotics; however, the benefits of olanzapine are offset by its propensity to cause significant weight gain, which may lead to long-term metabolic sequelae. Samidorphan is an opioid receptor antagonist that has mitigated olanzapine-induced weight gain in healthy adults and in adults with schizophrenia $[10,11]$. As stated in the Zyprexa US Package Insert [6], multiple doses of olanzapine did not affect the pharmacokinetics of lithium or valproate; therefore, dosage adjustment of lithium or valproate is not required with concomitant olanzapine administration. However, the potential effect of OLZ/SAM on the pharmacokinetics of lithium and valproate is unknown. Therefore, this study was conducted to assess the effects of multiple doses of OLZ/SAM on the pharmacokinetics of lithium or valproate.

\section{Methods}

\subsection{Study Design}

This was an open-label, single-sequence, two-cohort study (ALKS3831-B101) conducted at a single center in the USA (Vince and Associates Clinical Research, an Altasciences company, Overland Park, KS, USA). The study included two treatment cohorts (lithium cohort and valproate cohort) and two treatment periods (period 1, days 1-7; period 2, days 8-18). Lithium or valproate was administered as a monotherapy in period 1 (days 1-7) and concomitantly with OLZ/ SAM in period 2 (days 12-18). Blood samples for pharmacokinetic evaluation were collected prior to, and up to $12 \mathrm{~h}$ after, lithium or valproate dose administration on days 7 and 18. Subjects were admitted to the clinic on study day -1 and remained as inpatients for the duration of the study (Fig. 1).

In period 1 , subjects in the lithium cohort were administered lithium carbonate (referred to as lithium hereafter) $300-\mathrm{mg}$ tablets twice daily (600 mg/day), and subjects in the valproate cohort were administered divalproex sodium (referred to as valproate hereafter) 500-mg tablets twice daily (1000 mg/day) on days $1-6$. The twice-daily doses were administered after a morning and evening meal (separated by approximately $12 \mathrm{~h}$ ). On day 7 , only a single morning dose of lithium $300 \mathrm{mg}$ or valproate $500 \mathrm{mg}$ was administered approximately $12 \mathrm{~h}$ after the prior dose.

In period 2, OLZ/SAM (10 mg of olanzapine/10 $\mathrm{mg}$ of samidorphan) was administered once daily after a morning meal on days $8-18$. On days $12-17$, subjects in the lithium cohort resumed twice-daily doses of lithium $300 \mathrm{mg}$, and subjects in the valproate cohort resumed twice-daily doses of valproate $500 \mathrm{mg}$. On day 18, only a single morning dose of lithium $300 \mathrm{mg}$ or valproate $500 \mathrm{mg}$ was administered approximately $12 \mathrm{~h}$ after the prior dose. On days $12-18$, subjects received the morning dose of lithium or valproate concomitantly with the daily dose of OLZ/SAM. Trough (predose) concentrations of lithium or valproate were monitored on days $5,6,16$, and 17 . If any one of the trough concentrations was $>1.5 \mathrm{mEq} / \mathrm{L}$ for lithium or $>125 \mathrm{mg} / \mathrm{L}$ for valproate, that subject was discontinued from study treatment.

\subsection{Study Population and Restrictions}

Male and nonpregnant female volunteers, 18-40 years of age, who were in a good state of health confirmed by the absence of a clinically significant medical history or laboratory abnormalities were recruited. The use of prescribed or over-the-counter drugs, except for acetaminophen, multivitamins, or hormonal contraceptives, was not allowed. Products containing alcohol, caffeine, nicotine, and/or xanthines were disallowed for the duration of the study.

\subsection{Blood Sample Collection and Bioanalytical Methods}

Serial blood samples for characterizing pharmacokinetic profiles of lithium or valproate were collected prior to and at $0.25,0.5,1,1.5,2,4,8,10$, and $12 \mathrm{~h}$ after dosing of lithium or valproate on days 7 and 18. Plasma concentrations of lithium were analyzed by an independent laboratory (Syneos Health/inVentiv Health Clinique, Québec, 
QC, Canada) using a validated, inductively coupled, plasma mass spectrometry detection method. The assay has a calibration range of $20 \mathrm{ng} / \mathrm{mL}(0.00288 \mathrm{mEq} / \mathrm{L})$ to $10,000 \mathrm{ng} /$ $\mathrm{mL}(1.44 \mathrm{mEq} / \mathrm{L})$. Precision and accuracy of the method were evaluated by analyzing quality control (QC) samples at the lower limit of quantitation (LLOQ; $20.0 \mathrm{ng} / \mathrm{mL}$ ), low QC $(60.0 \mathrm{ng} / \mathrm{mL})$, medium QC (5000 ng/mL), and high QC $(7500 \mathrm{ng} / \mathrm{mL})$. The between-run precision [\% coefficient of variation $(\% \mathrm{CV})]$ ranged from 1.50 to $3.35 \%$ and the accuracy (\% bias) ranged from -0.88 to $0.54 \%$. Within-run precision (\%CV) ranged from 0.97 to $3.34 \%$ and the accuracy (\% bias) ranged from -4.03 to $2.20 \%$.

Plasma concentrations of valproic acid (referred to as valproate) were analyzed by another independent laboratory (Covance/Tandem, Salt Lake City, UT, USA) using a validated high-performance liquid chromatography with tandem mass spectrometric detection method. The assay has a calibration range of $0.200 \mu \mathrm{g} / \mathrm{mL}(0.200 \mathrm{mg} / \mathrm{L})$ to $100 \mu \mathrm{g} /$ $\mathrm{mL}(100 \mathrm{mg} / \mathrm{L})$. The LLOQ was $0.200 \mu \mathrm{g} / \mathrm{mL}(0.200 \mathrm{mg} / \mathrm{L})$ to $100 \mu \mathrm{g} / \mathrm{mL}(100 \mathrm{mg} / \mathrm{L})$. Dilution integrity was verified at a concentration up to $500 \mu \mathrm{g} / \mathrm{mL}$ when diluted ten-fold. Precision and accuracy of the method were evaluated by analyzing QC samples at the LLOQ $(0.200 \mu \mathrm{g} / \mathrm{mL})$, low QC $(0.600 \mu \mathrm{g} / \mathrm{mL})$, medium QC $(6.00 \mu \mathrm{g} / \mathrm{mL})$, and high QC $(75.00 \mu \mathrm{g} / \mathrm{mL})$. The between-run precision $(\% \mathrm{CV})$ ranged from 1.9 to $7.3 \%$ and the accuracy (\% bias) ranged from -1.5 to $3.5 \%$. Within-run precision ( $\% \mathrm{CV}$ ) ranged from 1.5 to $8.7 \%$ and the accuracy (\% bias) ranged from -4.0 to $3.8 \%$.

Blood samples for monitoring trough concentrations of lithium or valproate were collected within $1 \mathrm{~h}$ prior to dosing of lithium or valproate in the morning and evening on days 5, 6, 16, and 17. Trough concentrations of lithium or valproate were analyzed by a local laboratory in a timely manner as part of the safety monitoring. In addition, trough plasma concentrations of olanzapine and samidorphan were analyzed in blood samples collected within $1 \mathrm{~h}$ prior to dosing of OLZ/SAM on days 16, 17, and 18 using a validated liquid chromatography with tandem mass spectrometric method [12].

\subsection{Pharmacokinetic and Statistical Analyses}

Pharmacokinetic parameters for lithium or valproate, including maximum plasma concentration $\left(C_{\max }\right)$, time to $C_{\max }$ $\left(t_{\max }\right)$, and area under the plasma concentration-time curve over the 12-h dosing interval $\left(\mathrm{AUC}_{12 \mathrm{~h}}\right)$ were derived from concentration vs. time data on days 7 and 18. Pharmacokinetic parameters were calculated by a standard noncompartmental analysis method using Phoenix WinNonlin, version 7.0 (Pharsight, a Certara Company, Princeton, NJ, USA).

A mixed-effects model was used to analyze the natural log-transformed values of $C_{\max }$ and $\mathrm{AUC}_{12 \mathrm{~h}}$ for the intrasubject comparison of systemic exposures of lithium or valproate in the presence of OLZ/SAM (day 18) vs. in the absence of OLZ/SAM (day 7). The model included study treatment as a fixed effect and the subject as a random factor. The ratio of geometric means of $C_{\max }$ and $\mathrm{AUC}_{12 \mathrm{~h}}$ (in the presence vs. absence of OLZ/SAM) along with its twosided $90 \%$ confidence intervals (CIs) was estimated from the model. A lack of clinically significant effect of OLZ/SAM on the pharmacokinetics of lithium or valproate would be concluded if the $90 \%$ CIs for the ratios of geometric means, in the presence and absence of OLZ/SAM, were within the equivalence interval (i.e., no-effect boundary) of $80-125 \%$ for both $C_{\max }$ and $\mathrm{AUC}_{12 \mathrm{~h}}$ of lithium or valproate. All statistical calculations were performed with Statistical Analysis System (SAS), version 9.4 (SAS Institute Inc., Cary, NC, USA).

\subsection{Safety Assessments}

Safety was assessed based on vital signs and weight measurements, laboratory parameters (biochemistry, hematology, and urinalysis), and electrocardiogram recordings. The Columbia-Suicide Severity Rating Scale was administered at screening, day -1 , day 7 , day 18 , and at follow-up (day $25 \pm 3$ days). Adverse events (AEs) were monitored throughout the study.
Fig. 1 Study design schematic. Li lithium, OLZ/SAM olanzapine and samidorphan, Val val-

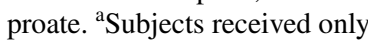
their morning dose of either lithium or valproate. ${ }^{\mathrm{b}} \mathrm{OLZ} /$ SAM 10/10 was administered in the morning on days 8-18

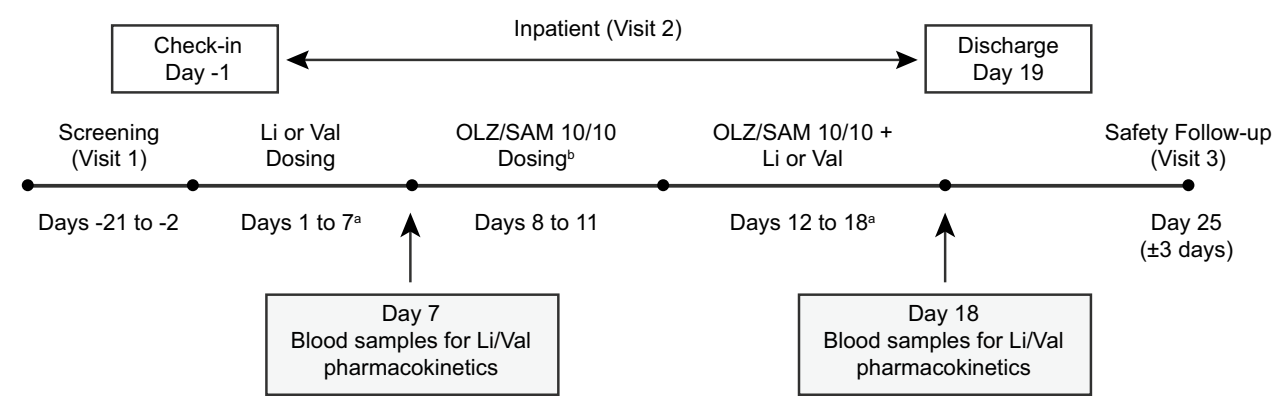

Period 1

Period 2 


\subsection{Ethical Approval}

The study was designed and carried out in accordance with the principles of Good Clinical Practice that have their origin in the Declaration of Helsinki and its amendments and in accordance with local regulations and International Conference on Harmonisation guidelines [13]. The study protocol and informed consent forms were approved by the clinical site's institutional review board (Midlands Independent Review Board, Overland Park, KS, USA) prior to any subjects being enrolled into the study. All subjects provided written informed consent before participating.

\section{Results}

A total of 34 healthy subjects were enrolled, 17 in the lithium cohort and 17 in the valproate cohort. All 17 subjects in the lithium cohort completed period 1 (lithium alone), and 16 (94.1\%) subjects completed period 2 (lithium plus OLZ/SAM). In the valproate cohort, 16 (94.1\%) subjects completed both period 1 (valproate alone) and period 2 (valproate plus OLZ/SAM). Mean age was 28.6 years in the lithium cohort and 33.0 years in the valproate cohort (Table 1). The majority of subjects were male (lithium cohort, $64.7 \%$; valproate cohort, 76.5\%) and black/African American (lithium cohort, 64.7\%; valproate cohort, 70.6\%). One subject in the lithium cohort discontinued in period 2 because of an AE of asymptomatic T-wave inversion in the anterior electrocardiogram leads that started approximately $24 \mathrm{~h}$ after dosing of OLZ/SAM on day 12 and resolved within $4 \mathrm{~h}$ on the same day. One subject in the valproate cohort discontinued from period 1 as a result of a high trough concentration of valproate $(132.6 \mathrm{mg} / \mathrm{L}$ prior to the evening dose on day $6)$, which exceeded the protocol-defined stopping criteria (>125 mg/L). The subject was asymptomatic; valproate concentration in a follow-up sample on day 8 was $73.2 \mathrm{mg} / \mathrm{L}$.

\subsection{Pharmacokinetics}

Trough concentrations of lithium and valproate on study days 5-7 (period 1) and days 16-18 (period 2) illustrated that steady-state concentrations of lithium and valproate were reached prior to dosing on day 7 (administered as monotherapy) and on day 18 (co-administered with OLZ/ SAM) (Fig. 2). In addition, trough concentrations of olanzapine and samidorphan on study days 16-18 illustrated that steady-state concentrations of olanzapine and samidorphan were also reached prior to co-administration with lithium or valproate on day 18 (Fig. 3).

Co-administration with OLZ/SAM did not have a notable effect on the steady-state pharmacokinetic profiles of lithium
(Fig. 4; Table 2). The 90\% CIs for the geometric mean ratios, in the presence vs. absence of OLZ/SAM, were within the equivalence interval of $80-125 \%$ for both $C_{\max }$ and $\mathrm{AUC}_{12 \mathrm{~h}}$ (Table 3), indicating that multiple-dose administration of OLZ/SAM to steady state did not have a clinically significant effect on the steady-state pharmacokinetics of lithium.

Similarly, co-administration with OLZ/SAM did not have a notable effect on the steady-state pharmacokinetic profiles of valproate (Fig. 5; Table 2). The 90\% CIs for the geometric mean ratios in the presence vs. absence of OLZ/SAM were within the 80-125\% equivalence interval for both $C_{\max }$ and $\mathrm{AUC}_{12 \mathrm{~h}}$ (Table 3), indicating that multiple-dose administration of OLZ/SAM to steady state did not have a clinically significant effect on the steady-state pharmacokinetics of valproate.

\subsection{Safety}

\subsubsection{Lithium Cohort}

A total of 8/17 (47.1\%) subjects reported at least one AE while receiving lithium only; all 17 subjects reported one or more AEs during treatment with lithium plus OLZ/SAM (Table 4). All AEs were mild or moderate in severity; no serious AEs or deaths were reported during either study period. The most common AEs (reported by three or more subjects) in the presence of OLZ/SAM were somnolence (17 subjects), dizziness (seven subjects), dry mouth (three subjects), and headache (three subjects). No AEs were reported by more than one subject in the absence of OLZ/SAM.

Three subjects developed AEs related to abnormal chemistry values; these included a blood creatine phosphokinase increase in two subjects (day 7, after lithium dosing; day 25, after lithium plus OLZ/SAM dosing), and an alanine aminotransferase increase in one subject (follow-up visit, after lithium plus OLZ/SAM dosing). Potentially clinically significant laboratory changes from baseline in period 1 included elevated fasting low-density lipoprotein cholesterol in two subjects and elevated fasting total cholesterol, elevated creatine kinase, or reduced fasting high-density lipoprotein cholesterol in one subject each. In period 2, elevated creatine kinase or alanine aminotransferase was observed in one subject each; elevated fasting triglyceride level was noted in one female and two male subjects. There were no clinically meaningful findings in hematology or urinalysis results other than a urinary tract infection in one subject that resolved. There were no clinically meaningful changes from baseline in vital signs, weight, or ColumbiaSuicide Severity Rating Scale measures in the lithium cohort. One subject had an AE of a T-wave inversion leading to study discontinuation. 
Table 1 Demographics and baseline characteristics

\begin{tabular}{lll}
\hline Characteristic & $\begin{array}{l}\text { Lithium } \\
\text { cohort }(n=17)\end{array}$ & $\begin{array}{l}\text { Valproate } \\
\text { cohort } \\
(n=17)\end{array}$ \\
\hline $\begin{array}{l}\text { Age, years } \\
\text { Mean (SD) }\end{array}$ & $28.6(5.21)$ & $33.0(4.64)$ \\
Range & $19-34$ & $24-40$ \\
Sex, $n(\%)$ & & \\
Female & $6(35.3)$ & $4(23.5)$ \\
Male & $11(64.7)$ & $13(76.5)$ \\
Ethnicity, $n(\%)$ & & \\
Hispanic or Latino & $2(11.8)$ & $2(11.8)$ \\
Not Hispanic or Latino & $15(88.2)$ & $15(88.2)$ \\
Race, $n(\%)$ & & \\
American Indian or Alaska Native & $2(11.8)$ & 0 \\
Black or African American & $11(64.7)$ & $12(70.6)$ \\
White & $4(23.5)$ & $5(29.4)$ \\
\hline
\end{tabular}

$S D$ standard deviation
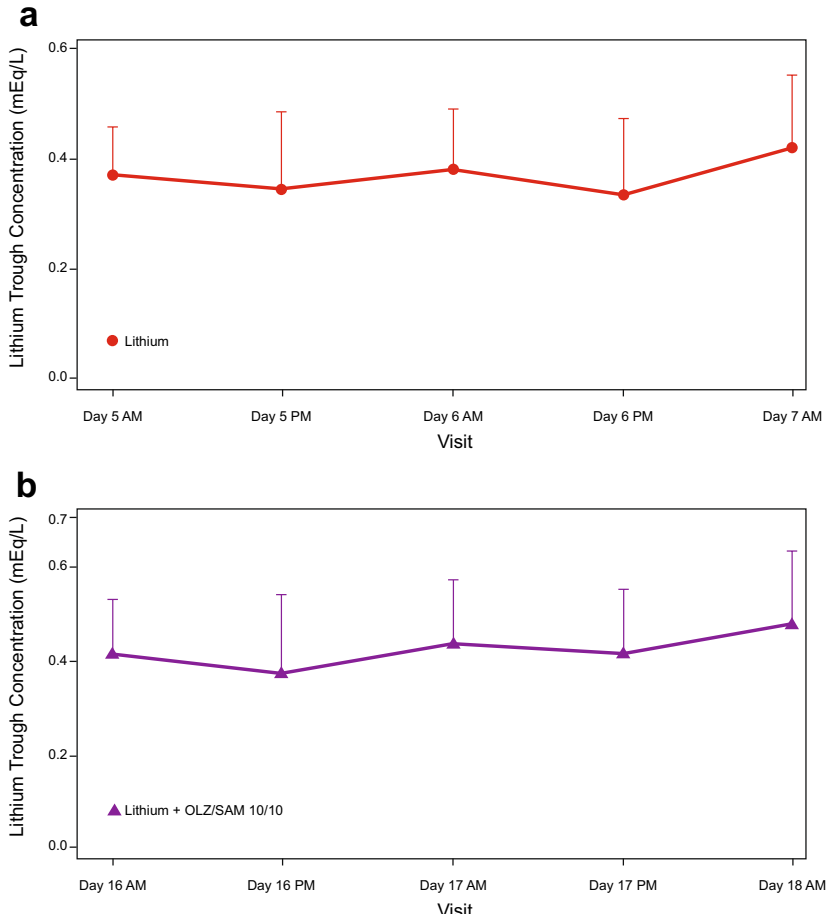

Fig. 2 Lithium and valproate trough concentrations (mean + standard deviation) when administered as monotherapy $(\mathbf{a}, \mathbf{c})$ or in combination with OLZ/SAM (10 mg of olanzapine/10 mg of samidorphan) (b, d). a Lithium concentration in period 1 (days 5-7); b lithium

\subsubsection{Valproate Cohort}

During exposure to valproate alone, $7 / 17$ (41.2\%) subjects reported one or more AEs; 16/16 subjects reported AEs throughout period 2 during exposure to OLZ/SAM and valproate (Table 4). All AEs were mild or moderate in severity, and no serious AEs or deaths occurred. The most common AEs in the presence of OLZ/SAM (reported by three or more subjects) were somnolence [16 (100\%) subjects] and dizziness [seven (43.8\%) subjects]. No AEs were reported by three or more subjects in the absence of OLZ/SAM.

In the valproate cohort, there were no clinically meaningful findings for serum chemistry, hematology, or urinalysis. In period 1, potentially significant changes from baseline in chemistry values included reductions in high-density lipoprotein cholesterol $(n=2)$ and elevations in triglycerides ( $n=2$ male subjects). In period 2, potentially significant changes included elevated creatine kinase, elevated lowdensity lipoprotein cholesterol, or reduced high-density lipoprotein cholesterol ( $n=1$ subject each) and elevated triglycerides in one female subject. There were no clinically meaningful changes from baseline in vital signs, weight, electrocardiogram, or Columbia-Suicide Severity Rating Scale measures in cohort 2.
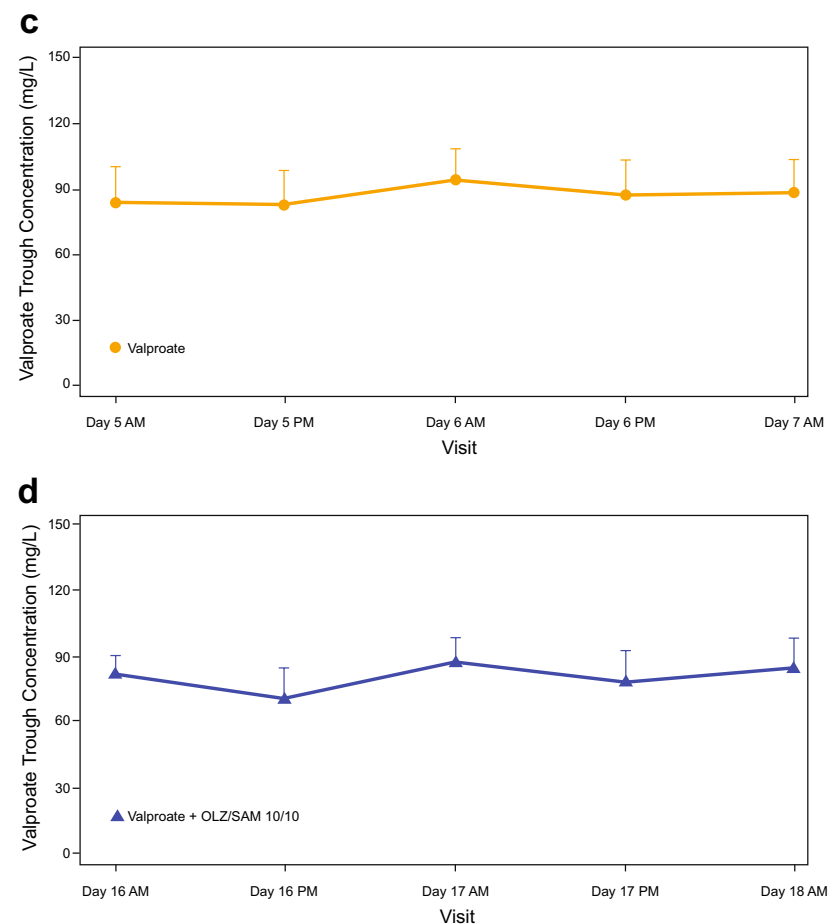

concentration in period 2 (days 16-18); $\mathbf{c}$ valproate concentration in period 1 (days 5-7); and $\mathbf{d}$ valproate concentration in period 2 (days 16-18) 
a

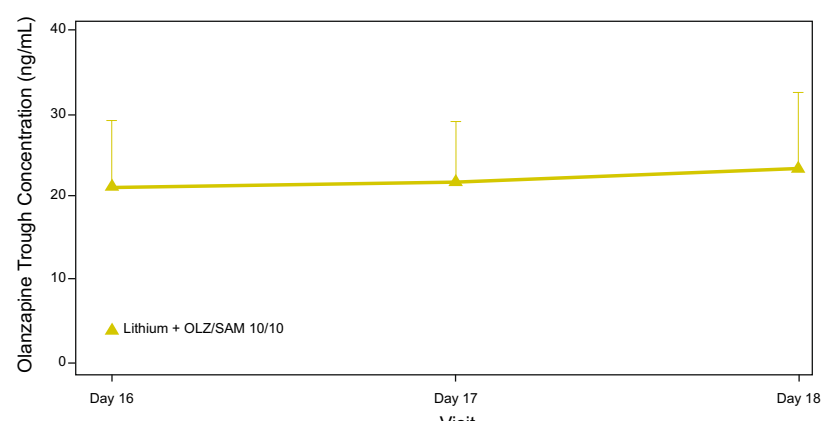

b

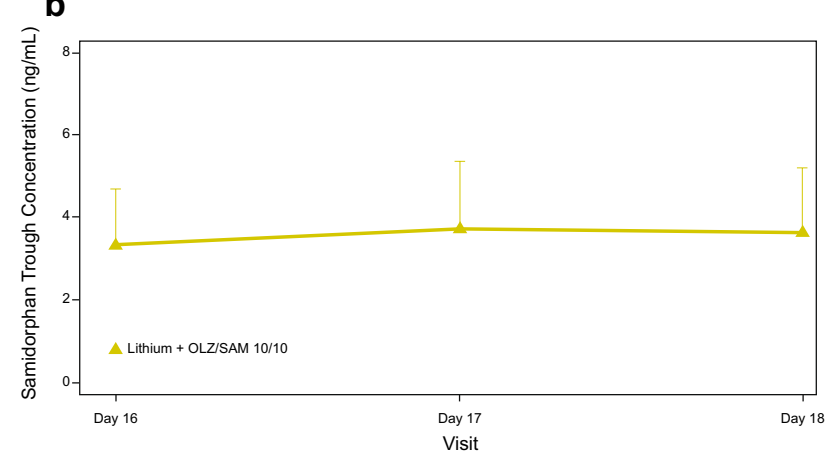

Fig. 3 Olanzapine and samidorphan (OLZ/SAM) trough concentrations [mean + standard deviation (SD)] on study days 16-18 from cohort 1 (lithium, a, b) and cohort 2 (valproate, c, d). a Olanzapine concentration in period 2 (days 16-18) in lithium cohort; b samidor-

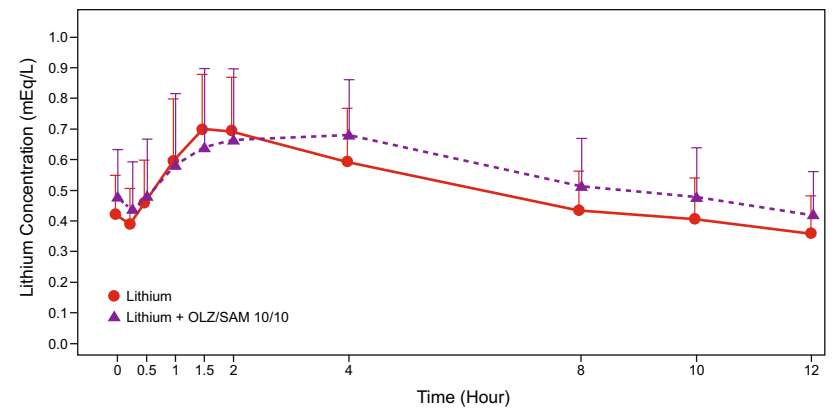

Fig. 4 Plasma concentrations [mean + standard deviation (SD)] of lithium following lithium $300 \mathrm{mg}$ administered alone and co-administered with $10 \mathrm{mg}$ of olanzapine/10 mg of samidorphan (OLZ/SAM)

\section{Discussion}

This study was designed to evaluate the effect of multipledose administrations of OLZ/SAM on the steady-state pharmacokinetics of lithium or valproate by comparing the exposures of lithium or valproate after seven daily administrations in the absence (day 7) and the presence (day 18) of OLZ/SAM. The additional days of OLZ/SAM-only dosing on days 8-11 were included to ensure steady-state
C
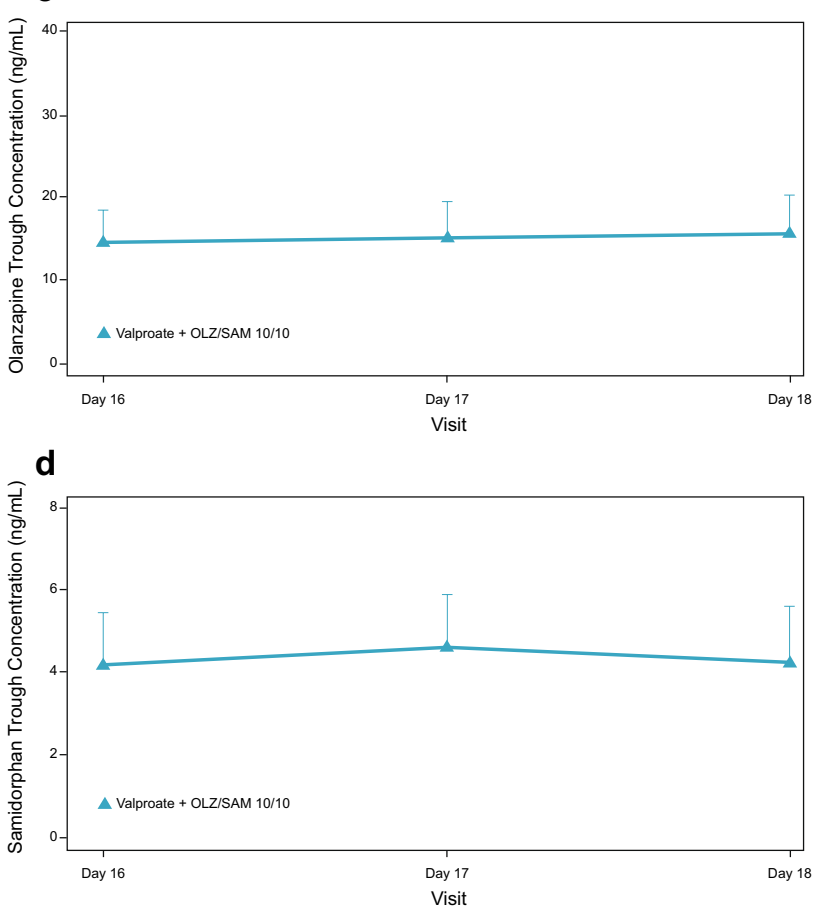

phan concentration in period 2 (days 16-18) in lithium cohort; c olanzapine concentration in period 2 (days 16-18) in valproate cohort; and $\mathbf{d}$ samidorphan concentration in period 2 (days 16-18) in valproate cohort

concentrations of olanzapine and samidorphan were reached prior to day 18 (given the long half-life of olanzapine [14]) to maximize the likelihood of detecting a potential effect of OLZ/SAM on the pharmacokinetics of lithium and valproate. Multiple-dose administration of OLZ/SAM to steady state did not have a clinically significant effect on the steady-state pharmacokinetics of lithium or valproate in this study. The $90 \%$ CIs of the geometric mean ratios of $C_{\max }$ and $\mathrm{AUC}_{12 \mathrm{~h}}$ for both lithium and valproate in the presence vs. absence of OLZ/SAM were within the equivalence interval of $80-125 \%$. These results were consistent with the findings previously reported for olanzapine, [6] and suggest that no dosage adjustment of lithium or valproate is necessary with concomitant use of OLZ/SAM. As illustrated by trough concentrations, steady-state concentrations of lithium and valproate were reached prior to day 7 and day 18, and steady-state concentrations of olanzapine and samidorphan were reached prior to day 18 .

Because long-term pharmacotherapy is required for the treatment of bipolar I disorder (with lithium or valproate recommended as first-line options) [15], the treatment effect and target drug concentrations of lithium or valproate are driven by steady-state exposure rather than acute exposure after a single dose. Therefore, the current study investigated 
Table 2 Summary of pharmacokinetic parameters for lithium or valproate in the absence and presence of olanzapine and samidorphan (OLZ/ SAM)

\begin{tabular}{|c|c|c|c|c|}
\hline \multirow[t]{2}{*}{ Parameter statistics } & Day 7 & Day 18 & Day 7 & Day 18 \\
\hline & $\begin{array}{l}\text { Lithium } 300 \mathrm{mg} \\
(n=17)\end{array}$ & $\begin{array}{l}\text { Lithium } 300 \mathrm{mg}+\text { OLZ/SAM } \\
(n=16)\end{array}$ & $\begin{array}{l}\text { Valproate } 500 \mathrm{mg} \\
(n=16)\end{array}$ & $\begin{array}{l}\text { Valproate } 500 \mathrm{mg}+\text { OLZ/SAM } \\
(n=16)\end{array}$ \\
\hline \multicolumn{5}{|l|}{$C_{\max }$} \\
\hline Mean (SD) & $0.733(0.173) \mathrm{mEq} / \mathrm{L}$ & $0.752(0.205) \mathrm{mEq} / \mathrm{L}$ & $103.0(18.6) \mathrm{mg} / \mathrm{L}$ & 89.8 (12.7) mg/L \\
\hline \multicolumn{5}{|l|}{$t_{\max }, \mathrm{h}$} \\
\hline Median (min, $\max )$ & $1.5(0.5,2.1)$ & $3.0(1.0,4.0)$ & $4.0(0.0,8.0)$ & $4.0(0.0,12.0)$ \\
\hline \multicolumn{5}{|l|}{$\mathrm{AUC}_{12 \mathrm{~h}}$} \\
\hline Mean (SD) & $6.09(1.75) \mathrm{mEq} \cdot \mathrm{h} / \mathrm{L}$ & $6.78(2.00) \mathrm{mEq} \cdot \mathrm{h} / \mathrm{L}$ & $1017.1(158.5) \mathrm{mg} \cdot \mathrm{h} / \mathrm{L}$ & $869.2(98.4) \mathrm{mg} \cdot \mathrm{h} / \mathrm{L}$ \\
\hline
\end{tabular}

$A U C_{12 h}$ area under the plasma concentration-time curve over the 12-h dosing interval, $C_{\max }$ maximum plasma concentration, $\max$ maximum, min minimum, $S D$ standard deviation, $t_{\max }$ time to $C_{\max }$

Table 3 Comparison of systemic exposures of lithium or valproate in the presence vs. absence of olanzapine and samidorphan (OLZ/SAM)

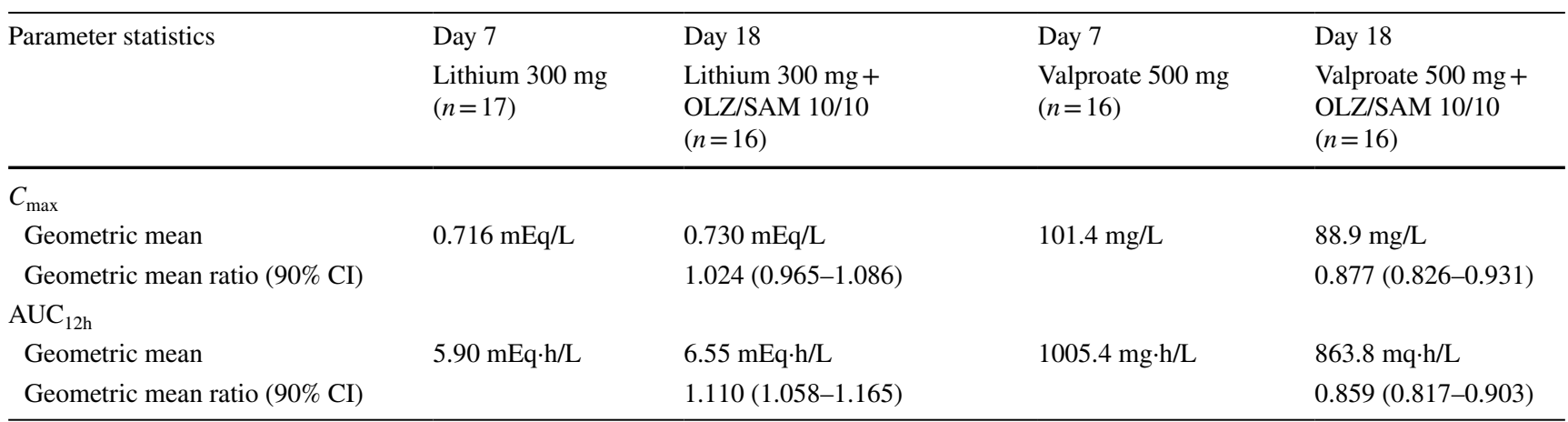

$A U C_{12 h}$ area under the plasma concentration-time curve over the 12 -h dosing interval, $C I$ confidence interval, $C_{\max }$ maximum plasma concentration

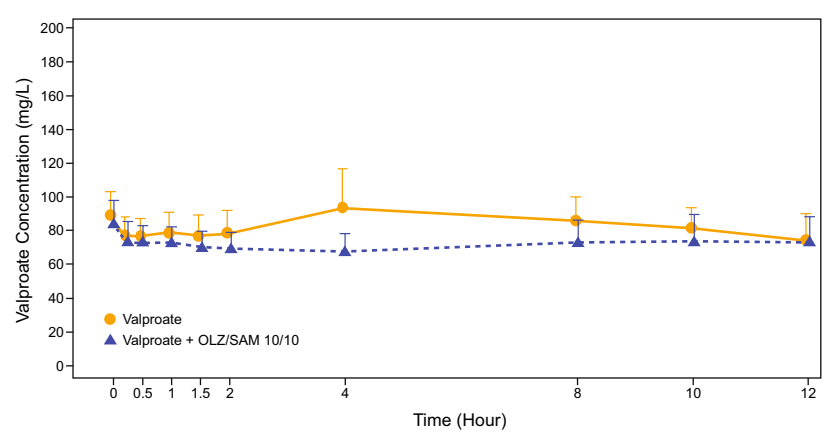

Fig. 5 Plasma concentrations [mean + standard deviation (SD)] of valproate following valproate $500 \mathrm{mg}$ administered alone and coadministered with $10 \mathrm{mg}$ of olanzapine/10 $\mathrm{mg}$ of samidorphan (OLZ/ SAM)

the effect of OLZ/SAM on steady-state pharmacokinetics of lithium or valproate.

The dose of OLZ/SAM (10 mg/10 mg) used in this study was clinically relevant, was expected to be generally well tolerated, and fell within the range of OLZ/SAM doses, 5/10 to $20 / 10 \mathrm{mg}$, under phase III clinical evaluation for efficacy and safety. In addition, the 10-mg olanzapine dose is within the recommended starting dose range for the treatment of mixed or manic episodes of bipolar I disorder when used either as monotherapy or as an adjunctive treatment to lithium or valproate [6].

Concomitant use of OLZ/SAM is not expected to have an impact on the pharmacokinetics of lithium and valproate owing to their divergent metabolic and elimination pathways. Olanzapine is mainly eliminated via hepatic metabolism, with $7 \%$ of the administered dose being excreted renally as unchanged olanzapine [16]. The primary metabolic pathways for olanzapine are direct glucuronidation via uridine 5'-diphospho-glucuronosyltransferase (UGT) 1A4 and cytochrome P450 (CYP)-mediated oxidation, mainly by CYP1A2 with minor contributions from other CYP enzymes, including CYP2C8, CYP3A4, and CYP2D6 $[16,17]$. Samidorphan is eliminated primarily via CYP3A4mediated hepatic metabolism with approximately $20 \%$ of the dose being excreted renally as unchanged samidorphan [18]. Lithium is not hepatically metabolized and is excreted 
Table 4 Number of subjects reporting adverse events (AEs) in the absence and in the presence of olanzapine and samidorphan (OLZ/ SAM) (AEs reported by one or more subject in the lithium or valproate cohorts in either period: safety population)

\begin{tabular}{llllll}
\hline AEs, $n(\%)$ & \multicolumn{2}{l}{$\begin{array}{l}\text { Lithium cohort } \\
(600 \mathrm{mg} / \text { day })\end{array}$} & & \multicolumn{2}{l}{$\begin{array}{l}\text { Valproate cohort } \\
\text { (1000 mg/day })\end{array}$} \\
\cline { 2 - 3 } \cline { 6 - 7 } & $\begin{array}{l}\text { Period 1 } \\
\text { Days 1-7 } \\
(n=17)\end{array}$ & $\begin{array}{l}\text { Period 2 } \\
\text { Days 8-25 } \\
(n=17)\end{array}$ & & $\begin{array}{l}\text { Period 1 } \\
\text { Days 1-7 } \\
(n=17)\end{array}$ & $\begin{array}{l}\text { Period 2 } \\
\text { Days 8-25 } \\
(n=16)\end{array}$ \\
\hline Any AE & $8(47.1)$ & $17(100)$ & & $7(41.2)$ & $16(100)$ \\
Somnolence & $1(5.9)$ & $17(100)$ & & $1(5.9)$ & $16(100)$ \\
Dizziness & $1(5.9)$ & $7(41.2)$ & & $1(5.9)$ & $7(43.8)$ \\
Headache & $1(5.9)$ & $3(17.6)$ & $2(11.8)$ & $2(12.5)$ \\
Dry mouth & 0 & $3(17.6)$ & 0 & $1(6.3)$ \\
Constipation & 0 & $2(11.8)$ & 0 & $1(6.3)$ \\
Fatigue & $1(5.9)$ & $2(11.8)$ & 0 & $1(6.3)$ \\
Feeling abnormal & 0 & $2(11.8)$ & 0 & 0 \\
Tremor & 0 & $2(11.8)$ & 0 & $2(12.5)$ \\
\hline
\end{tabular}

Period 1: On study days 1-6, subjects were given oral doses of $300 \mathrm{mg}$ of lithium (lithium cohort) or $500 \mathrm{mg}$ of valproate (valproate cohort 2) twice daily (separated by approximately $12 \mathrm{~h}$ ) after the morning and evening meals. On study day 7 , a single dose of lithium or valproate was administered after the morning meal (approximately $12 \mathrm{~h}$ after the prior dose)

Period 2: On study days 8-18, subjects were administered once-daily oral doses of OLZ/SAM 10/10 (10 mg of olanzapine/10 mg of samidorphan) each morning after the morning meal. On study days 12-17, subjects resumed lithium (lithium cohort) or valproate (valproate cohort) twice daily (separated by approximately $12 \mathrm{~h}$ ) after the morning and evening meals. Subjects received the morning dose of lithium or valproate concomitantly with the daily dose of OLZ/SAM 10/10 (10 mg of olanzapine/10 $\mathrm{mg}$ of samidorphan)

almost entirely renally [19]; therefore, it is unlikely to be a victim drug in drug-drug interactions with olanzapine or samidorphan. Valproate is extensively metabolized hepatically, predominantly by glucuronidation, accounting for $50 \%$ of valproate disposition. The remainder undergoes beta-oxidation ( $40 \%$ ) mediated by beta-oxidative enzymes in the mitochondria and CYP-mediated oxidation (10\%) [19]. Glucuronidation, a major metabolic pathway of valproate, is mediated by UGTs, mainly UGT1A4 [20]; CYP-mediated oxidation, mainly by CYP2C9, is a minor pathway [21]. Valproate is also unlikely to be a victim in drug-drug interactions with olanzapine or samidorphan, as hepatic CYPmediated oxidation is a minor metabolic pathway [21], and there is little evidence that it is a substrate for P-glycoprotein [22]. Valproate is a substrate for CYP2C9 and CYP2C19 [23], but neither olanzapine nor samidorphan significantly inhibits or induces CYP2C9 or CYP2C19 activity [14, 24].

Although the median $t_{\max }$ of lithium observed on day 18 with co-administration of OLZ/SAM ( $3 \mathrm{~h}$ ) was slightly delayed compared with the median $t_{\max }$ observed on day 7 with lithium alone $(1.5 \mathrm{~h})$, the ranges of $t_{\max }$ observed in the two dosing periods (Table 2) were overlapping and were consistent with the range of $t_{\max }(0.5-3 \mathrm{~h})$ previously reported for lithium [25]. Moreover, co-administration with OLZ/SAM did not have a clinically significant effect on the $C_{\max }$ and $\mathrm{AUC}_{12 \mathrm{~h}}$ of lithium, and therefore the slight difference in median $t_{\max }$ of lithium observed between co-administration with OLZ/SAM and lithium alone is not considered clinically meaningful.

Co-administration of OLZ/SAM with lithium or valproate was generally well tolerated in this study, and the safety and tolerability profile of OLZ/SAM was consistent with findings from previous OLZ/SAM studies in healthy adults [10, 12]. For both lithium and valproate cohorts, $\mathrm{AE}$ rates were higher in the presence of OLZ/SAM; all subjects reported one or more AEs in period 2, during exposure to OLZ/SAM. Adverse events reported in the presence of OLZ/SAM were generally similar to the AE profile for olanzapine administered alone [26]. Overall, the most common AEs in period 2 (reported by three or more subjects in either cohort) were somnolence, dizziness, dry mouth, and headache and coincided with initiation of OLZ/SAM treatment. Co-administration of OLZ/SAM and lithium or valproate on study day 12 and thereafter did not result in the increased incidence or severity of the AEs evaluated and did not appear to extend their duration, as the majority of AEs resolved prior to the completion of period 2 . The safety profiles of lithium or valproate in this study were consistent with their known safety and tolerability profiles [27, 28]. In addition, the safety profiles of lithium or valproate co-administered with OLZ/SAM were consistent with what has been previously reported [27, 28].

\section{Conclusions}

Co-administration of OLZ/SAM did not have a clinically significant effect on the steady-state pharmacokinetics of lithium or valproate. Co-administration of OLZ/SAM with lithium or valproate was generally well tolerated, and AEs that occurred during OLZ/SAM exposure were consistent with those observed in other clinical studies of OLZ/SAM in healthy subjects, with no notable increase in severity or duration during co-administration. Based on these results, no dosage adjustment of lithium or valproate would be necessary when co-administered with OLZ/SAM.

Acknowledgements The authors thank Mark S. Todtenkopf, PhD, who assisted in the preparation and proofreading of the manuscript. Medical writing and editorial support was provided by Kathleen M. Dorries, Ph.D., and John H. Simmons, M.D., of Peloton Advantage, LLC, an OPEN Health company, and funded by Alkermes, Inc. 


\section{Compliance with Ethical Standards}

Funding This study was sponsored by Alkermes, Inc., which is a pharmaceutical company developing OLZ/SAM, a combination product of olanzapine and samidorphan for the treatment of schizophrenia.

Conflict of interest Lei Sun, Sergey Yagoda, Baiyun Yao, Christine Graham, and Lisa von Moltke are employees of Alkermes, Inc.

Ethics approval The study was designed and carried out in accordance with the principles of Good Clinical Practice that have their origin in the Declaration of Helsinki and its amendments and in accordance with local regulations and International Conference on Harmonisation guidelines. The study protocol and informed consent forms were approved by the clinical site's institutional review board prior to any subjects being enrolled into the study.

Informed consent All subjects provided written informed consent before participating in the study.

Open Access This article is distributed under the terms of the Creative Commons Attribution-NonCommercial 4.0 International License (http://creativecommons.org/licenses/by-nc/4.0/), which permits any noncommercial use, distribution, and reproduction in any medium, provided you give appropriate credit to the original author(s) and the source, provide a link to the Creative Commons license, and indicate if changes were made.

\section{References}

1. The global burden of disease: 2004 update. World Health Organization. http://www.who.int/healthinfo/global_burden_disea se/2004_report_update/en/index.html. Accessed 27 June 2019.

2. Hirschfeld RMA, Bowden CL, Gitlin MJ, et al. Practice guideline for the treatment of patients with bipolar disorder. 2nd ed. Washington, DC: American Psychiatric Association; 2002.

3. Goodwin GM. Evidence-based guidelines for treating bipolar disorder: revised second edition-Recommendations from the British Association for Psychopharmacology. J Psychopharmacol (Oxford). 2009;23:346-88.

4. Yatham LN, Kennedy SH, Parikh SV, et al. Canadian Network for Mood and Anxiety Treatments (CANMAT) and International Society for Bipolar Disorders (ISBD) collaborative update of CANMAT guidelines for the management of patients with bipolar disorder: update 2013. Bipolar Disord. 2013;15:1-44.

5. Fountoulakis KN, Grunze H, Vieta E, et al. The International College of Neuro-Psychopharmacology (CINP) Treatment Guidelines for Bipolar Disorder in Adults (CINP-BD-2017), Part 3: the clinical guidelines. Int J Neuropsychopharmacol. 2017;20:180-95.

6. Zyprexa [package insert]. Indianapolis: Eli Lilly and Company; 2018.

7. Cipriani A, Barbui C, Salanti G, et al. Comparative efficacy and acceptability of antimanic drugs in acute mania: a multiple-treatments meta-analysis. Lancet. 2011;378:1306-15.

8. Grunze H, Vieta E, Goodwin GM, et al. The World Federation of Societies of Biological Psychiatry (WFSBP) guidelines for the biological treatment of bipolar disorders: acute and long-term treatment of mixed states in bipolar disorder. World J Biol Psychiatry. 2018;19:2-58.

9. Cipriani A, Rendell JM, Geddes J. Olanzapine in long-term treatment for bipolar disorder. Cochrane Database Syst Rev. 2009;1:CD004367.
10. Silverman BL, Martin W, Memisoglu A, et al. A randomized, double-blind, placebo-controlled proof of concept study to evaluate samidorphan in the prevention of olanzapine-induced weight gain in healthy volunteers. Schizophr Res. 2018;195:245-51.

11. Martin WF, Correll CU, Weiden PJ, et al. Mitigation of olanzapine-induced weight gain with samidorphan, an opioid antagonist: a randomized double-blind phase 2 study in patients with schizophrenia. Am J Psychiatry. 2019;176:457-67.

12. Sun L, McDonnell D, von Moltke L. Pharmacokinetics and shortterm safety of ALKS 3831, a fixed-dose combination of olanzapine and samidorphan, in adult subjects with schizophrenia. Clin Ther. 2018;40:1845-54.

13. International Conference on Harmonisation of Technical Requirements for Registration of Pharmaceuticals for Human Use (ICH). E6(R2). Guideline for good clinical practice. European Medicines Agency; 2002. http://www.ema.europa.eu/docs/en_GB/docum ent_library/Scientific_guideline/2009/09/WC500002874.pdf. Accessed 27 June 2019.

14. Callaghan JT, Bergstrom RF, Ptak LR, Beasley CM. Olanzapine: pharmacokinetic and pharmacodynamic profile. Clin Pharmacokinet. 1999;37:177-93.

15. Yatham LN, Kennedy SH, Parikh SV, et al. Canadian Network for Mood and Anxiety Treatments (CANMAT) and International Society for Bipolar Disorders (ISBD) 2018 guidelines for the management of patients with bipolar disorder. Bipolar Disord. 2018;20:97-170.

16. Kassahun K, Mattiuz E, Nyhart E Jr, et al. Disposition and biotransformation of the antipsychotic agent olanzapine in humans. Drug Metab Dispos. 1997;25:81-93.

17. Korprasertthaworn P, Polasek TM, Sorich MJ, et al. In vitro characterization of the human liver microsomal kinetics and reaction phenotyping of olanzapine metabolism. Drug Metab Dispos. 2015;43:1806-14.

18. Sun L, McDonnell D, Yu M, Kumar V, von Moltke L. A phase I open-label study to evaluate the effects of rifampin on the pharmacokinetics of olanzapine and samidorphan administered in combination in healthy human subjects. Clin Drug Investig. 2019;39(5):477-84

19. Ketter TA, Frye MA, Cora-Locatelli G, Kimbrell TA, Post RM. Metabolism and excretion of mood stabilizers and new anticonvulsants. Cell Mol Neurobiol. 1999;19:511-32.

20. Argikar UA, Remmel RP. Effect of aging on glucuronidation of valproic acid in human liver microsomes and the role of UDPglucuronosyltransferase UGT1A4, UGT1A8, and UGT1A10. Drug Metab Dispos. 2009;37:229-36.

21. Spina E, Pisani F, de Leon J. Clinically significant pharmacokinetic drug interactions of antiepileptic drugs with new antidepressants and new antipsychotics. Pharmacol Res. 2016;106:72-86.

22. Zhang C, Kwan P, Zuo Z, Baum L. The transport of antiepileptic drugs by P-glycoprotein. Adv Drug Deliv Rev. 2012;64:930-42.

23. Schoretsanitis G, Haen E, Grunder G, et al. Pharmacokinetic drug-drug interactions of mood stabilizers and risperidone in patients under combined treatment. J Clin Psychopharmacol. 2016;36:554-61.

24. Ring BJ, Catlow J, Lindsay TJ, et al. Identification of the human cytochromes $\mathrm{P} 450$ responsible for the in vitro formation of the major oxidative metabolites of the antipsychotic agent olanzapine. J Pharmacol Exp Ther. 1996;276:658-66.

25. Ward ME, Musa MN, Bailey L. Clinical pharmacokinetics of lithium. J Clin Pharmacol. 1994;34:280-5.

26. Bhana N, Foster RH, Olney R, Plosker GL. Olanzapine: an updated review of its use in the management of schizophrenia. Drugs. 2001;61:111-61.

27. Lithium [package insert]. Columbus: Roxane Laboratories, Inc.; 2016.

28. Depakote [package insert]. North Chicago: AbbVie Inc.; 2017. 


\section{Affiliations}

\section{Lei Sun ${ }^{1} \cdot$ Sergey Yagoda ${ }^{2}$ Baiyun $\mathrm{Yao}^{3} \cdot$ Christine Graham $^{2} \cdot$ Lisa von Moltke}

1 Department of Clinical Pharmacology and Translational Medicine, Alkermes, Inc., 852 Winter Street, Waltham, MA 02451, USA

2 Department of Clinical Research, Alkermes, Inc., 852 Winter Street, Waltham, MA 02451, USA
3 Department of Biostatistics, Alkermes, Inc., 852 Winter Street, Waltham, MA 02451, USA 